\title{
Anatomía del fruto de Casimiroa edulis (Rutaceae), "zapote blanco", durante su desarrollo
}

\author{
Hilda Araceli Zavaleta-Mancera ${ }^{1,2}$ y E. Mark ENGleman ${ }^{1}$
}

\begin{abstract}
RESUMEN. El zapote blanco es un fruto mexicano apreciado por su pulpa dulce comestible y por sus semillas medicinales. Tomando en cuenta la importancia de esta especie para México y la escasa información sobre la anatomía del fruto, se propuso estudiar el desarrollo del fruto desde antesis hasta madurez comestible. Se hicieron cortes y disociaciones. Se aplicaron tinciones generales con safranina y verde fijo, y particulares para almidón, lípidos, fenoles (taninos) y lignina. El crecimiento en diámetro del fruto presenta una curva simple sigmoide. La estructura fibrosa que cubre la semilla es un endocarpo que se origina de a) la epidermis interna pluriestratificada, b) estratos subepidérmicos de tejido fundamental y c) una red de tejido vascular que rodea el lóculo. Las paredes de este tejido se engruesan y lignifican poco antes de la madurez. El pericarpo no acumula almidón durante el desarrollo; en la madurez es dulce y con abundantes esferosomas (gotitas de lípidos); contiene numerosas glándulas de aceite de origen lisígeno, de formas y tamaños $(0.1-5.0 \mathrm{~mm})$ variables, cuyos ejes se disponen radialmente en el fruto. En el material estudiado no observamos la hipodermis externa descrita por Schroeder.
\end{abstract}

ABSTRACT. White sapote is a Mexican fruit valued for its edible sweet pulp and medicinal seeds. In view of the importance that this species has for Mexico, and considering the scarcity of information on the anatomy of its fruit, a study of its development from flowering to maturity was undertaken. The material was studied by sectioning and maceration. General and staining with safranin and fast green, as well as specific staining for starch, lipids, phenols (tannins) and lignin, were made. The fruit growth in diameter presents a simple sigmoid growth curve. The fibrous structure covering the seed is an endocarp originating from

${ }^{1}$ Centro de Botánica, Colegio de Postgraduados, 56230 Montecillo, México.

${ }^{2}$ Este trabajo representa parte de la tesis de Maestro en Ciencias, presentada por la primera autora en 1989 en el Colegio de Postgraduados, Montecillo, México. 


\begin{abstract}
a) the multiple internal epidermis, b) subepidermal layers of fundamental tissue and c) a net of vascular tissue surrounding the locule. The walls of this tissue thicken and lignify just before maturity. The pericarp does not accumulate starch during growth; at maturity it is sweet and contains abundant spherosomes (lipid droplets). The pericarp is populated by numerous lysigenous oil glands presenting various shapes and sizes $(0.1-5.0 \mathrm{~mm})$, with their axes radially oriented. We did not find the external hypodermis reported by Schroeder.
\end{abstract}

Casimiroa edulis Llave et Lexarza, comunmente llamado zapote blanco, pertenece a la subfamilia Toddalioideae de la familia Rutaceae. Es nativa de México y se encuentra en zonas subtropicales y templadas hasta la altitud de $2500 \mathrm{~m}$, en forma silvestre, escapada o dentro del huerto familiar (Popenoe, 1920; Martínez, 1951 y Ochse, et al, 1965). Entre las casimiroas, C. edulis y C. sapota Oerst. son las más conocidas y cultivadas, porque ofrecen frutos grandes y comestibles. En Florida y California se han seleccionado ya algunas variedades de C. edulis (William y Harold, 1960 y Morton, 1962).

El fruto es globoso o ligeramente alargado, de color verde amarillento, liso, de 6-10 $\mathrm{cm}$ de diámetro. El exocarpo es delgado, de sabor amargo. El mesocarpo (pulpa), es blanco o algo amarillento, de consistencia cremosa, de sabor dulce y algo indigesto (Martínez, 1951). El fruto maduro posee de uno a cinco huesos coriáceos que contienen una semilla ovoide de 3-5 cm de largo y 2-3 cm de ancho, sin endospermo (Engler, 1931; Schroeder, 1954 y Martínez, 1951).

Casimiroa edulis no sólo es apreciada por sus frutos dulces y comestibles sino también por sus propiedades medicinales. Bajo el nombre de "cochitzápotl" el fruto fue usado por los mexicas como alimento y somnífero (Códice Florentino, 1980, escrito entre 1560 y 1580). Actualmente se sabe que los extractos acuosos y alcohólicos de las hojas y semillas producen un efecto similar a los de la histamina, entre ellos bajar la presión arterial (Lozoya, et al., 1977).

A pesar de que C. edulis se ha clasificado dentro de la subfamilia Toddalioideae, por considerar que el fruto es una drupa, algunos autores como Martínez (1951) y Bickerm (1897, citado por Netolitzky, 1926) y Corner (1976) se refieren a la estructura fibrosa que cubre la semilla como testa. Engler (1931) y Schroeder (1954) la refieren como endocarpo. Hartl (1958), menciona que los endocarpos de Toddalioideae se originan exclusivamente de la epidermis locular o interna.

Se conocen principalmente dos tipos de crecimiento en diámetro de frutos carnosos, la curva simple sigmoide y la curva doble sigmoide. La curva doble sigmoide presenta una detención intermedia en su crecimiento y la simple sigmoide no (Coombe, 1976 y Bollard, 1970).

Con este estudio se pretende conocer y analizar el desarrollo del fruto y estudiar la ontogenia del tejido protector de la semilla, para cotejar los resultados obtenidos con la literatura. 


\section{MATERIALES Y MÉTODOS}

1. Colecta. Los lugares en donde se hicieron observaciones y colectas fueron: a) Huerto familiar propiedad del Sr. Armando Domínguez, localizado en San Luis Huexotla, Estado de México, altitud $2270 \mathrm{~m}$, a $19^{\circ} 28^{\prime} 42^{\prime \prime} \mathrm{N}-98^{\circ} 52^{\prime} 14^{\prime \prime} \mathrm{W}$. b) Cerco vivo propiedad de la Sra. Delgadina Leyva localizado en La Garita, Jungapeo, Michoacán, altitud 1520 m, a $19^{\circ} 28^{\prime} 50^{\prime \prime} \mathrm{N}-100^{\circ} 29^{\prime} 14^{\prime \prime} \mathrm{W}$.

A partir de enero de 1987 se hicieron algunas observaciones y colectas preliminares en tres árboles de Huexotla. De estas colectas se estudió la anatomía de algunas flores y frutos. Posteriormente, de diciembre de 1987 a mayo de 1988 se colectaron flores y frutos en un árbol de La Garita. Los muestreos se hicieron: uno el 20 de diciembre de 1987, y siete en 1988, en las siguientes fechas; 9 y 30 de enero, 20 de febrero, 13 y 27 de marzo, 23 de abril y 8 de mayo. En el primer muestreo, además de frutos jóvenes, se colectaron flores tardías que aún existían en el árbol. También el 5 y 26 de noviembre de 1988 se hicieron algunas observaciones adicionales en La Garita para conocer el fenómeno completo de la floración.

Griggs e Iwakeri en 1956 (citados por Bollard, 1970) apuntan que hay una correspondencia íntima entre las curvas de crecimiento obtenidas de medir frutos cosechados a intervalos de tiempo y de medir frutos in situ. No obstante, cuando la floración es errática, o hay mucha variación entre plantas, es recomendable medir los mismos frutos in situ a lo largo del desarrollo. Cuando los árboles son altos e inaccesibles y hay riesgo del ataque de plagas o enfermedades, como fue el caso de nuestro estudio, el evaluar muestras a intervalos de tiempo es más práctico y seguro que trabajar con frutos marcados que pueden perderse.

En cada fecha de colecta se cortaron aproximadamente de 30 a 70 frutos, de las ramas ubicadas en los primeros $6 \mathrm{~m}$. Una escalera de tijeras y una garrocha ayudaron a alcanzar los frutos más altos. Siempre se cortaron los frutos más grandes en ese momento. Luego los frutos fueron cortados transversalmente y se seleccionaron únicamente los frutos con dos semillas normales, debido a que en frutos de la misma edad sus diámetros varían, dependiendo del número de semillas que desarrollan (los frutos con dos y tres semillas fueron los más abundantes, por esta razón se decidió trabajar con frutos de dos semillas). Posteriormente los frutos con dos semillas se agruparon en clases, tomando como referencia sus diámetros ecuatoriales. Los intervalos para cada clase se establecieron en una secuencia logarítmica. Así, cada intervalo o clase guardó una relación de 0.75 con el intervalo subsecuente (tabla 1).

2. Curva de crecimiento. En cada fecha de muestreo se registró cuáles clases estaban presentes. De las encontradas, se completaron 10 frutos por clase. En estos frutos se midió el diámetro ecuatorial y se calculó el logaritmo base 10 de dichos valores. Con el promedio de los logarítmos de los diámetros de los frutos seleccionados (10, 20 ó 30) en 
cada fecha, se elaboró la curva de crecimiento. Con la ayuda de esta gráfica se estimó el período de floración, así como la edad en días de cada clase (fig. 1).

3. Microtecnia. Algunos botones y frutos jóvenes se fijaron en FAA ( $10 \%$ formalina $+48 \%$ de etanol $+5 \%$ ácido acético $+37 \%$ agua), los frutos restantes se fijaron en partes iguales de Craf III solución A (23\% formalina en agua) y Craf III solución B (0.6 $\%$ trióxido de cromo $+4 \%$ ácido acético en agua). Después los tejidos se deshidrataron en series graduales de etanol para ser incluidos en parafina. Los cortes de 8 a 10 um se tiñeron con safranina 0 y verde fijo FCF o con rojo 0 de aceite $(0.05 \% \mathrm{p} / \mathrm{v}$ rojo 0 de aceite $+25 \% \mathrm{v} / \mathrm{v}$ de 1 -butanol $+75 \% \mathrm{v} / \mathrm{v}$ etilenglicol). En cortes de tejido fresco o previamente conservado en etanol se hicieron tinciones para: lípidos con rojo 0 de aceite; almidón con solución saturada de yodo $+0.5 \%$ de yoduro de potasio; lignina agregando dos gotas de $(2 \%$ floroglucinol en etanol $)$ y dos gotas de $(30 \% \mathrm{HCl})$ sobre el corte; proteínas con (1\% azul negro de naftol en etanol $50 \%$ ); leucoantocianinas y catequinas agregando dos gotas de (10\% vanillina en etanol) y luego dos gotas de $(50 \% \mathrm{HCl}) ;$ fenoles con solución ácida de cloruro férrico $(1 \%$ cloruro férrico en $1 \mathrm{~N} \mathrm{HCl})$; disociación con solución Jeffrey ( $9 \%$ de trióxido de cromo $+10 \%$ ácido nítrico concentrado). Las referencias bibliográficas de las anteriores técnicas se citan en Zavaleta-Mancera y Engleman (1991).

4. Crecimiento del pericarpo. En el exocarpo, mesocarpo y cuerpo central se evaluó para cada clase: a) el diámetro celular, b) presencia o ausencia de figuras mitóticas y paredes recién formadas, c) presencia o ausencia y tamaño cualitativo de los espacios intercelulares. El exocarpo se definió como la epidermis externa más los primeros ocho estratos subepidérmicos; el mesocarpo como el tejido fundamental entre exocarpo y endocarpo; el cuerpo central como el tejido del centro del fruto, rodeado por los cinco lóculos y el endocarpo como la capa de células de pared gruesa que rodea cada lóculo.

\section{RESULTADOS}

En Huexotla C. edulis floreció en enero y febrero. Los meses de floración coincidieron con los meses más frios del año cuando comúnmente hay heladas. En consecuencia hubo pérdidas considerables de flores y frutos. Los cortes hechos de flores y frutos jóvenes en Huexotla coicidieron anatómicamente con los de La Garita. Porque el segưndo sitio ofreció más material y mejores datos de edad y tamaño, la siguiente descripción se basa en el árbol de La Garita.

1. Observaciones de campo. En La Garita, C. edulis floreció en noviembre y diciembre. La ausencia de heladas favoreció el desarrollo normal de flores y frutos jóvenes. El período de desarrollo (antesis a madurez) de los frutos aquí estudiados fue de aproximadamente 175 días.

Se observó que muchos de los frutos colectados en La Garita estaban infestados por larvas; las cuales consumieron preferentemente el embrión de la semilla. Las larvas se 


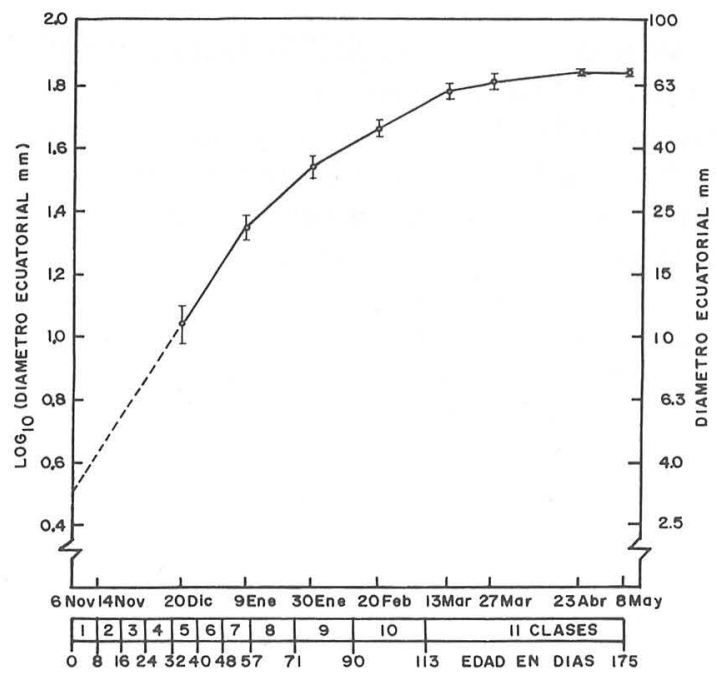

Fig. 1. Curva de crecimiento de frutos con dos semillas de C. edulis. En cada fecha de colecta, el diámetro registrado se basa en el promedio de un número determinado de los frutos pertenecientes a las clases encontradas ( $\mathrm{n}=10,20$ ó v 30) \pm el error estándar de la media. Ver el texto para la extrapolación de la curva hacia la floración.

TABLA 1. Definición de las clases de diámetro ecuatorial de los frutos. El límite inferior incluye el valor y ell límite superior lo excluye.

Clase

Diámetro ecuatorial, $\mathrm{mm}$ 
observaron claramente a partir de la clase 10 (90-113 días), cuando la semilla alcanzó el $30 \%$ de su volumen final y el embrión rico en almidón ocupaba todo el volumen de la semilla. Esta larva fue identificada como la mosca Anastrepha sp.

2. Curva de crecimiento. En la figura 1 la línea punteada extrapola la curva con la misma pendiente que hay entre los puntos correspondientes a las colectas del 20 de diciembre de 1987 y 9 de enero de 1988. Esta proyección se usó para estimar la edad de las clases 1 hasta 4. Así se calculó que del 6 al 14 de noviembre de 1987 aproximadamente, el árbol se encontraba en plena floración, tomando como referencia el diámetro ecuatorial del ovario. Esto concordó con las observaciones realizadas en noviembre de 1988. La mayor tasa relativa de crecimiento se observó entre el 20 de diciembre de 1987 y 9 de enero de 1988. Luego decreció progresivamente hasta detenerse a los 160 días. El tamaño promedio que alcanzaron los frutos maduros con dos semillas fue de $66 \mathrm{~mm}$ (fig. 1).

Puesto que la curva se presenta con escala logarítmica, una línea recta representa una tasa relativa de crecimiento constante. Si esta misma curva se gráficara en escala aritmética, tendría la forma sigmoide simple.

3. Anatomía del pericarpo.

3.1 Endocarpo. El endocarpo del fruto es una estructura fibrosa y dura que cubre cada semilla (figs. 2,3 ); tiene un grosor de 0.7 a $1.5 \mathrm{~mm}$ y proviene de tres renglones de la pared del ovario (fig. 5):

1) Epidermis interna o locular pluriestatificada.

2) Tejido fundamental subepidérmico.

3) Red de tejido vascular que rodea el lóculo, siguiendo direcciones, más o menos paralelas al eje del fruto.

La superficie interna del endocarpo (figs. 3,5,6) es lisa y lustrosa; está compuesta principalmente por fibras orientadas en direcciones horizontal y oblicua (fig. 6). Las fibras miden en promedio $740 \mu$ de largo y $16 \mu$ de ancho; los lúmenes son amplios y la pared es de aproximadamente $4 \mu$ de grosor, con punteaduras simples.

La porción externa del endocarpo (figs. 3,5,6) proviene de la red de tejido vascular. En la madurez está compuesta de fibras y esclereidas (fig. 4), la mayoría tiende a orientarse paralelamente al eje principal del fruto (figs. 3, 6). Las fibras son similares a las ya descritas pero más largas; miden en promedio $990 \mu$ de largo y $18 \mu$ de ancho. Las esclereidas (fig. 4) pueden ser isodiaméticas, cónicas, angulosas o alargadas. La variación en tamaño va de 20 a $310 \mu$ de largo y de 20 a $40 \mu$ de diámetro. La pared varía de 4 a $6 \mu$ de grosor. En el tejido vascular se pueden encontrar algunos elementos de vaso punteados y otros con engrosamientos helicoidales o anulares. Las placas de perforación son simples o escalariformes (fig. 4).

Las paredes celulares del futuro endocarpo empiezan a engrosarse cuando el fruto tiene entre 42 y $56 \mathrm{~mm}$ de diámetro (clase 10). El endocarpo se lignifica una vez que el fruto alcanza su tamaño final (clase 11, fig. 6), justo antes de que la pulpa se ablande. 
Primero se lignifica la región interna ubicada junto al lóculo, luego la región próxima a la pulpa.

3.2 Exocarpo. El exocarpo del fruto maduro (figs. 2,7) es la parte que se desprende del fruto al pelarlo. Está formado por:

1) La epidermis externa, la cual posee algunos tricomas y numerosos estomas. Esta epidermis siempre se divide anticlinalmente y está cubierta por una cutícula que aumenta durante el desarrollo de 1-2 $\mu$ a 10-17 $\mu$ de grosor (fig. 7).

Los tricomas son escasos, pero están presentes desde la antesis hasta poco antes de la madurez comestible del fruto. Son pelos simples de dos a cuatro células. Son muy frágiles, y sólo pudieron observarse completos en cortes a mano de frutos recién traídos del campo. La cutícula y tricomas se tiñeron intensamente con rojo 0 de aceite.

2) Tejido subepidérmico de 6-8 estratos de células pequeñas, alargadas tangencialmente, con pocos taninos (detectados con vanillina y $\mathrm{HCl}$ ) y numerosos cloroplastos. Los cloroplastos tienen granos de almidón. Este tejido contiene braquiesclereidas aisladas o en grupos de dos a tres, y numerosas glándulas de aceite, usualmente esféricas. Las esclereidas (fig. 7) se diferenciaron entre los 90 y 113 días (clase 10).

3.3 Mesocarpo. El mesocarpo, o pulpa, está formado por el parénquima ubicado entre el exocarpo y el endocarpo (fig. 2). Se caracteriza por la presencia de células grandes de paredes delgadas (fig. 9), espacios intercelulares y numerosas glándulas de aceite de origen lisígeno (fig. 2).

En la antesis la pared del ovario ya tiene algunas glándulas de aceite pero la mayoría está en proceso de diferenciación. Durante el desarrollo estas cavidades secretoras aumentan en número y tamaño. Las glándulas localizadas cerca de la epidermis externa generalmente son pequeñas y esféricas; las más internas adoptan una diversidad de tamaños (0.1-5.0 mm de largo) y formas (esféricas, piriformes, alargadas, constreñidas en el centro, con apéndices). Desde la antesis hasta la madurez los ejes de las glándulas alargadas tienen una orientación radial en la pulpa.

El parénquima normal de la pulpa nunca almacenó almidón. Sin embargo este parénquima sí acumuló lípidos durante todo el desarrollo, principalmente durante la maduración del fruto. Los lípidos de la pulpa se presentaron como esferosomas o pequeñas gotitas de lípidos disgregadas. De las tinciones para proteína y fenoles no se encontró ninguna respuesta positiva relevante.

Durante los primeros 40 días las células eran pequeñas (21 a $28 \mu)$, se dividían mucho y carecían de espacios intercelulares (fig. 8). De los 40 a los 113 días las células se ensancharon y siguieron dividiéndose. En este momento los espacios intercelulares empezaron a formarse. En la última etapa del crecimiento del fruto, no hubo evidencia de división celular; las células aumentaron su diámetro a $70 \mu$ en promedio (fig. 9, 10), y los espacios intercelulares se hicieron más grandes. Entre las clases 9 a 11, las células del cuerpo central y del exocarpo aumentaron poco en diámetro; en el mismo período las células del mesocarpo aumentaron en un 50\% (fig. 10). 


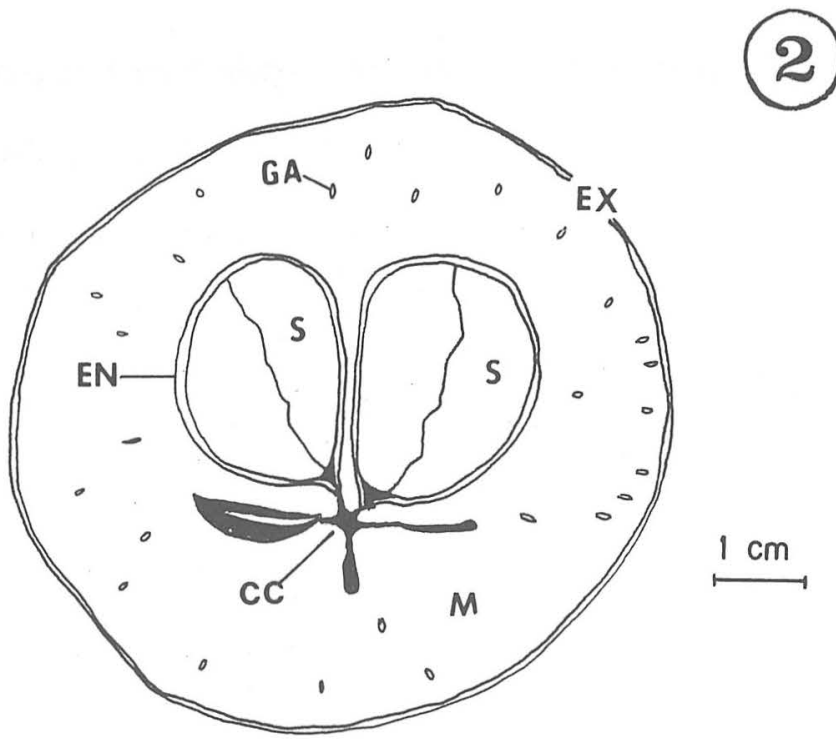

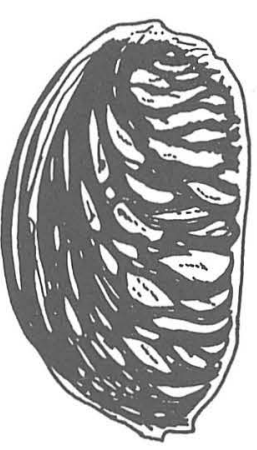

A
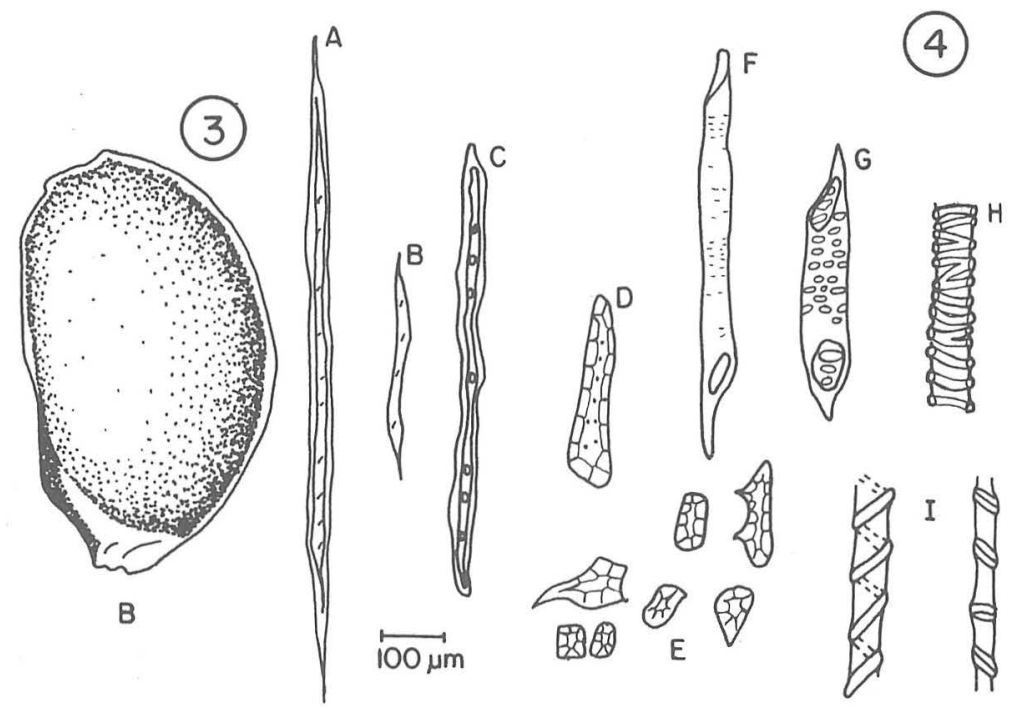

Fig. 2. Corte transversal de un fruto maduro; ex = exocarpo; $m=$ mesocarpo; en = endocarpo; ga = glándula de aceite; $\mathrm{s}=$ semilla; $\mathrm{cc}=$ cuerpo central.

Fig. 3. Endocarpo de un fruto maduro. $\mathrm{A}=$ vista externa; $\mathrm{B}=$ vista interna.

Fig. 4. Células lignificadas del endocarpo. A, B, C = fibras; D, E = esclereidas; F, G, H, I = miembros de vaso. 


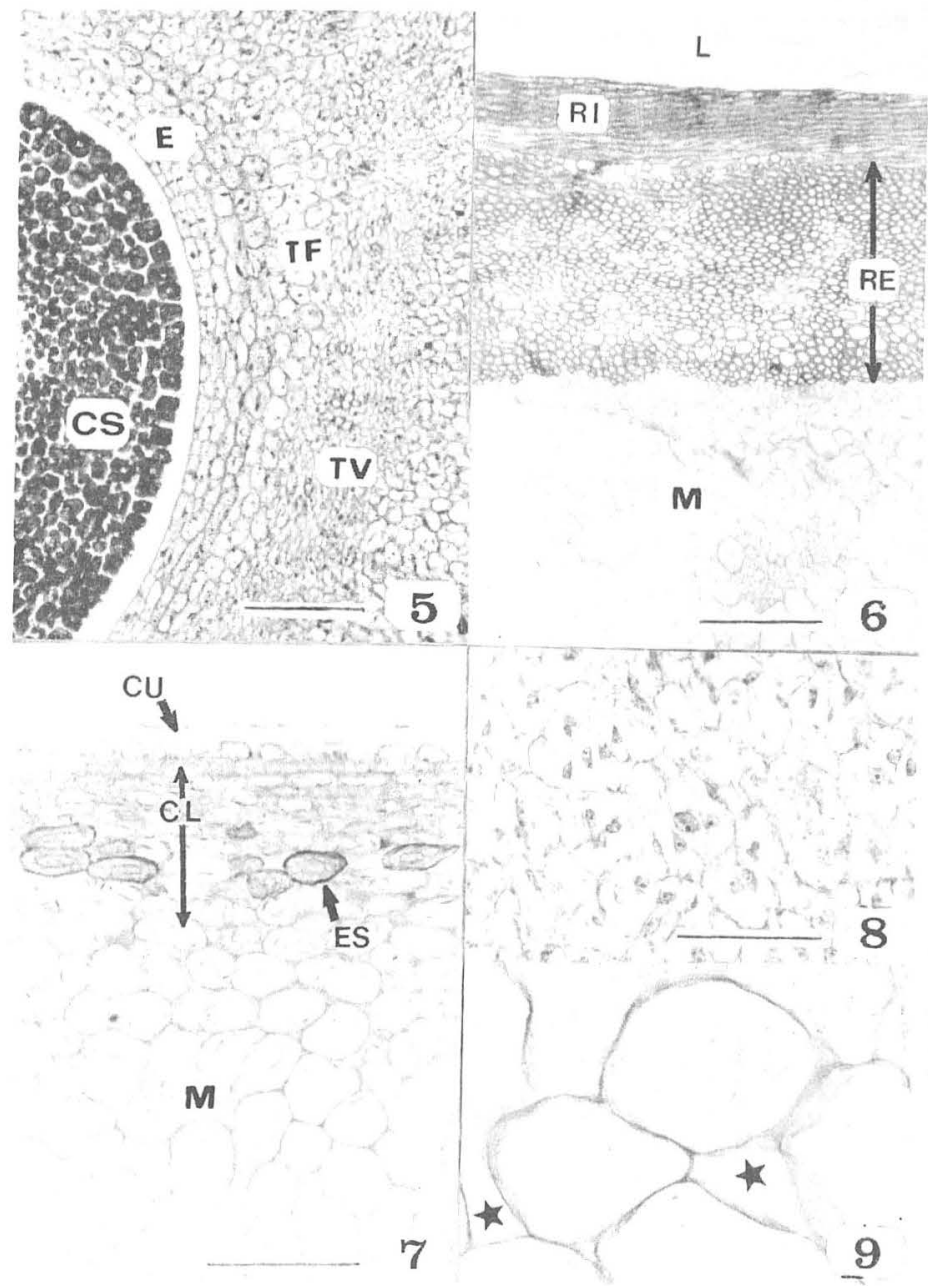

Fig. 5. Tejido precursor del endocarpo en corte transversal del fruto (Clase 1), E = Epidermis locular pluriestratificada con 3-4 estratos; TF = Tejido fundamental subepidérmico; $\mathrm{TV}=$ Tejido vascular; $\mathrm{CS}$ $=$ Cubierta seminal. Barra $=100 \mu$

Fig. 6. Endocarpo maduro totalmente lignificado (clase 11) en corte transversal, RI = Región interna; RE = Región externa; $\mathrm{L}=$ Lóculo; $\mathrm{M}=$ Mesocarpo. Barra $=100 \mu$

Fig. 7. Corte transversal del exocarpo maduro. Nótese el grosor de la cutícula (CU). ES = Esclereida; CL = Clorénquima; $\mathrm{M}=$ Mesocarpo. Barra $=100 \mu$

Fig. 8. Corte transversal del mesocarpo (clase 1). Nótese el citoplasma denso. Barra $=50 \mu$ Fig. 9. Corte transversal del mesocarpo maduro. Las estrellas indican los espacios intercelulares. Barra = $50 \mu$ 
BOLETIN DE LA SOCIEDAD BOTANICA DE MEXICO, Núm. 51, 1991

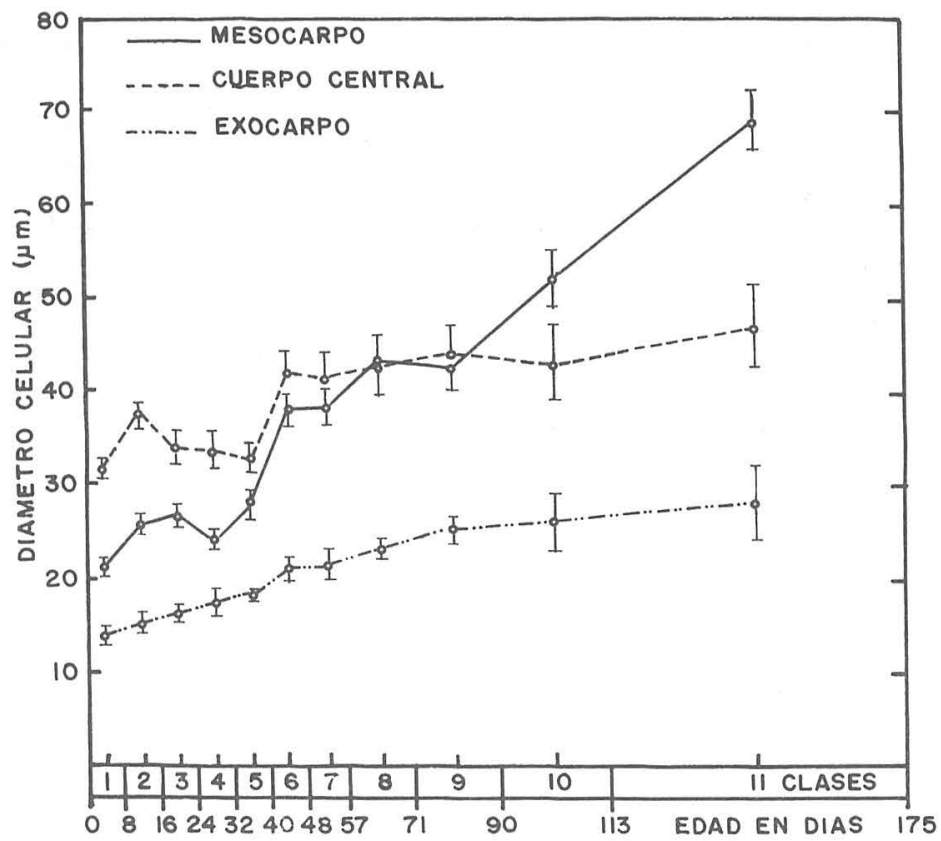

Fig. 10. Diámetro celular del mesocarpo, cuerpo central y exocarpo de C. edulis durante el desarrollo. Los valores son promedios de 16 células de cuatro frutos por clase \pm el error estándar de la media.

3.4 Cuerpo central. El cuerpo central (fig. 2) corresponde al tejido que queda en el eje central del fruto. En el corte transversal del fruto maduro, el cuerpo central es un tejido esclerenquimático en forma de una estrella, cuyos vértices apuntan hacia el hilo de cada semilla. Se caracteriza por la formación temprana de espacios intercelulares y la diferenciación de braquiesclereidas de 40 a $130 \mu$ de diámetro hacia la madurez, al mismo tiempo cuando se lignifica el endocarpo.

\section{DISCUSIÓN Y CONCLUSIONES}

En la literatura se mencionan dos patrones de crecimiento de frutos carnosos (medidos en diámetro, volumen y peso fresco), la curva simple sigmoide y la doble sigmoide. La doble sigmoide presenta dos fases de crecimiento activo separado por un período de crecimiento nulo o escaso. Esta curva se ha encontrado principalmente en frutos con hueso duro como el durazno (Prunus persica), ciruela (Prunus domestica) y cereza (Prunus cerasus) según Tukey en 1934 (citado por Roth, 1977). En estas especies se han observado tres períodos, I, II y III, donde los componentes del fruto (mesocarpo, endocarpo y semilla) no se desarrollan simultáneamente. Durante el período I el fruto 
se agranda rápidamente y los tegumentos de la semilla se desarrollan al mismo tiempo, mientras que el embrión permanece pequeño. En el período II el embrión acelera su crecimiento, mientras que el fruto disminuye o detiene su crecimiento. A medida que el crecimiento del embrión se detiene, el fruto entra de nuevo en un período de crecimiento activo, el cual constituye el período III detallado por Tukey en 1933 (citado por Nitsch 1953 y Bollard 1970).

Se tiene la idea de que el crecimiento se detiene cuando el endocarpo endurece y el embrión crece rápidamente Lilleland en 1930 (citado por Roth, 1977) y Tukey en 1933 y 1934 (citado por Bollard, 1970).

Hay otros frutos como el higo (Ficus carica), frambuesa (Rubus idaeus), arándano (Vaccinium sp.) y uva con semillas (Vitis vinifera) que también presentan una curva doble sigmoide (Bollard, 1970).

Al revisar la bibliografía se oberva que el comportamiento doble sigmoide no es exclusivo de drupas; por esta razón, las hipótesis que se dan para explicar este comportamiento en Prunus no pueden generalizarse. Creemos que para proponer hipótesis nuevas para explicar el crecimiento del fruto de $C$. edulis deben tomarse en cuenta las siguientes características:

1) Las curvas de crecimiento de la semilla y embrión (Zavaleta-Mancera y Engleman 1991) y del fruto son simples sigmoides.

2) El endocarpo se engruesa y endurece (lignifica) hacia el final del desarrollo cuando el fruto ha alcanzado su tamaño final.

3) El endocarpo de C. edulis es menos grueso y duro que el endocarpo de Prunus spp.

Según Nitsch (1970), el desarrollo del fruto depende del desarrollo de la semilla y en consecuencia el peso final del fruto generalmente es proporcional al número de semillas que se desarrollan. Para calcular la edad con base en el diámetro del fruto, aquí fue necesario muestrear frutos del mismo número de semillas para eliminar la variable mencionada por Nitsch. Así hubo una marcada relación entre el diámetro y la edad del fruto, hecho que se reflejó en la uniformidad estructural que se observó dentro de cada intervalo de diámetro.

Schroeder (1954) describe una hipodermis de aproximadamente tres células de grosor debajo de la epidermis externa de frutos de la variedad Pike, provenientes de la huerta del Departamento de Horticultura Subtropical de la Universidad de California (USA). Sin embargo, en los frutos de La Garita no se observó dicha hipodermis. Tendría que revisarse otras poblaciones de Casimiroa edulis para conocer mejor la variación al respecto.

El período de división celular de C. edulis abarca las primeras 10 a 12 semanas. Este período es largo comparado con los períodos de división celular de muchos otros frutos (higo, manzana, frambuesa, ciruela, durazno. albaricoque, y otros), cuyos períodos de división celular no rebasan las 6 semanas después de la floración (Bollard, 1970). 
Si se observa la gráfica de diámetro celular del mesocarpo contra tiempo (fig. 10), la pendiente de la curva durante el período de división celular (clase 1-9) no indica precisamente la tasa de alargamiento en las paredes, ya que después de una división, las células hijas son pequeñas. Estas requieren primero alcanzar el tamaño de la célula madre, y luego rebasar este tamaño. A partir de la clase 10 , cuando no hay división celular, la pendiente de la curva sí indica la tasa absoluta de extensión de las paredes (micrómetros por día).

La ausencia de almidón durante el desarrollo del fruto y la abundancia de azúcares (Schroeder, 1954) y lípidos en el fruto maduro, sugieren que sería interesante estudiar el metabolismo del pericarpo.

El estudio de la diferenciación de la estructura fibrosa que cubre la semilla madura nos permitió identificarla como endocarpo en acuerdo con Engler (1931) y Schroeder (1954) y en oposición a Bickerm en 1903 (citado por Netolitzky, 1926) y Corner (1976). En estas circunstancias el fruto de zapote blanco debe considerarse como una drupa.

Los endocarpos de Skimmia (Toddallioideae) Hartl (1958) y Casimiroa edulis son semejantes en cuanto a la disposición horizontal y oblicua de las fibras adyacentes al lóculo, pero diferentes en cuanto a su origen, composición y grosor. El endocarpo de Skimmia es más delgado, se origina únicamente de la epidermis interna y está compuesto exclusivamente de fibras. Contrariamente, el endocarpo de $C$. edulis se origina de la epidermis interna y estratos subepidérmicos y está compuesto de fibras, esclereidas y elementos de vaso. Según Hartl (1958) los endocarpos de Toddalioideae se originan exclusivamente de la epidermis interna o locular. Esta generalización ya no es válida; hace falta revisar bien a otros miembros de la subfamilia.

Según Roth (1977) la presencia de fibras en el endocarpo puede considerarse un carácter primitivo, y la presencia de esclereidas como avanzado. Bajo este esquema, $C$. edulis ocuparía una posición filogenéticamente intermedia.

AGRADECIMIENTOS. Los autores agradecemos a la Sra. Delgadina Leyva y al Sr. Armando Domínguez por permitirnos muestrear y hacer observaciones en árboles de su propiedad. Al Consejo Nacional de Ciencia y Tecnología (CONACYT) por la beca de maestría otorgada a la primera autora. A Benjamín Zavaleta Rodríguez y Humberto López Delgado por su ayuda en el trabajo de campo. Al Centro de Entomología y Acarología del Colegio de Postgraduados por la identificación de las larvas de mosca y a un revisor anónimo de este Boletín por sus valiosas observaciones que ayudaron a mejorar el trabajo. 


\section{LITERATURA CITADA}

BOLLARD, G.E. 1970. The physiology and nutrition of developing fruit. En: Iulme, A.C. The biochemistry of fruits and their products. Academic Press, London. v.1:387-425.

CODICE FLORENTINO. 1980. Edición facsímil del manuscrito 218-20 de la Colección Palatina de la Biblioteca Medicea Laurenziana. Gobierno de la República Mexicana. 3v.

CoOMBE, B.G. 1976. The development of fleshy fruits. Annu. Rev. P1. Physiol. 27:207-228.

CORNER, E.J.H. 1976. The seeds of dicotyledons. Cambridge Univ. Press, London. 2v.

ENGLER, A. 1931. Rutaceae. En: Engler, A. y K. Prantl. Die natürlichen Pflanzenfamilien. 2a. ed. Band 19a:187-359.

GARDNER, R.O. 1975. Vanillin-hydrochloric acid as histochemical test for tannin. Stain. Technol. 50(5):315-317.

HARTL. D. 1958. Struktur und herkunft des endokarps der Rutaceen. Beitr. Biol. Pflanzen 34:35-49.

LEOPOLD, C.A. y E.P. KRIEDEMANN. 1975. Plant growth and development. 2a. ed. McGraw-Hill, New York. 545p.

LozoyA, X., G. Romero, M. OlmEdo y A. BondANI. 1977. Farmacodinámica de los extractos alcohólico y acuoso de la semilla de Casimiroa edulis. Arch. Invest. Méd. (México) 8:145-150.

MARTÍNEZ, M. 1951. Las casimiroas de México y Centroamérica. An. Inst. Biol. Univ. Nac. México 22(1):25-81.

MORTON, J. 1962. The drug of the white sapote. Econ. Bot. 16:288-294.

NeTOLITZKY, F. 1926. Anatomie der Angiospermen-Samen. Handbuch der Pflanzenanatomie. Borntraeger, Berlin. 364p.

NITSCH, J.P. 1953. The physiology of fruit growth. Annu. Rev. Plant Physiol. 4:199-236.

NITSCH, J.P. 1970. Hormonal factors in growth and development. En: Hulme, A.C. The biochemistry of fruits and their products. Academic Press, London. v.2:427-471.

OCHSE, J.J., M.J. JOULE, y J.M. DIJKMAN. 1965. Cultivo y mejoramiento de plantas tropicales y subtropicales. LIMUSA, México. 2v.

POPENOE, W. 1920. Manual of tropical and subtropical fruits. Hafner Press (Collier), New York. 474p.

ROTH, I. 1977. Fruits of angiosperms. Handbuch der Pflanzenanatomie. Borntraeger, Stuttgart. 675p.

SCHROEDER, C.A. 1954. Fruit morphology and anatomy in white sapote. Bot. Gaz. 115:248-254.

WilliaM, C.R. y E. HAROLD. 1960. Some fruits and nuts for the tropics. Agricultural Research Service, United States Department of Agriculture, Washington, D.C. Misc. Publ. No. 18. 135p.

ZavaleTA-MANCERA, H.A. y E.M. ENGLEMAN. 1991. Anatomía de la semilla de Casimiroa edulis (Rutaceae), "zapote blanco", durante su desarrollo. Bol. Soc. Bot. México. 51:67-81. 